\title{
Thermal conductivity of diamond and related materials from molecular dynamics simulations
}

\author{
Jianwei Che, Tahir Çağın, Weiqiao Deng, and William A. Goddard III \\ Materials and Process Simulation Center, Beckman Institute, \\ Division of Chemistry and Chemical Engineering, California Institute of Technology, Pasadena, \\ California 91125
}

(Received 25 January 2000; accepted 25 July 2000)

\begin{abstract}
Based on the Green-Kubo relation from linear response theory, we calculated the thermal current autocorrelation functions from classical molecular dynamics (MD) simulations. We examined the role of quantum corrections to the classical thermal conduction and concluded that these effects are small for fairly harmonic systems such as diamond. We then used the classical MD to extract thermal conductivities for bulk crystalline systems. We find that (at $300 \mathrm{~K}$ ) ${ }^{12} \mathrm{C}$ isotopically pure perfect diamond has a thermal conductivity $45 \%$ higher than natural $\left(1.1 \%{ }^{13} \mathrm{C}\right)$ diamond. This agrees well with experiment, which shows a 40\%-50\% increase. We find that vacancies dramatically decrease the thermal conductivity, and that it can be described by a reciprocal relation with a scaling as $n_{v}^{-\alpha}$, with $\alpha=0.69 \pm 0.11$ in agreement with phenomenological theory ( $\alpha=1 / 2$ to $3 / 4)$. Such calculations of thermal conductivity may become important for describing nanoscale devices. As a first step in studying such systems, we examined the mass effects on the thermal conductivity of compound systems, finding that the layered system has a lower conductivity than the uniform system. (C) 2000 American Institute of Physics. [S0021-9606(00)70140-1]
\end{abstract}

\section{INTRODUCTION}

As the dimensions of electronic and mechanical devices are shrunk into nanometer dimensions, the thermal conductivity becomes quite important since functioning electronic, piezoelectric, and thermogalvanic devices may require that significant energy be dissipated in a small region. However, the experimental measurement of thermal conductivity ${ }^{1}$ becomes quite difficult for nanometer scale devices, particularly for the complex geometries of real devices. Consequently, it is of interest to develop reliable theoretical and computational methods for predicting the thermal properties of nanoscale materials and devices.

There are two major approaches to theoretical studies of the thermal conductivities of materials.

(1) The most fundamental approach is to base the calculations on first principles atomistic simulations. This allows the properties for new materials to be predicted in advance of experiment. This is particularly useful for nanoscale devices where the experiments are quite difficult. Atomistic simulations have been employed to determine diffusion coefficients, viscosities, and thermal conductivities for fluids. Both equilibrium and nonequilibrium dynamic simulations ${ }^{2-4}$ have been reported for various systems. The calculated results are often in reasonable agreement with experimental measurements.

(2) More commonly, thermal conductivity has been studied using continuum models and kinetic theories such as the Boltzmann transport equation. For example, Walkauskas et al. ${ }^{5}$ calculated the lattice thermal conductivity of GaAs

\footnotetext{
a) Author to whom all correspondence should be addressed; electronic mail: wag@wag.caltech.edu
}

wires; Balandin and Weng ${ }^{6}$ calculated the thermal conductivity reduction in the $\mathrm{Si}$ quantum well; Gurevich ${ }^{7}$ discussed in detail the use of general principles of physical kinetics to describe phonon transport theory. These approaches must assume the fundamental constitutive equation and parameters.

The advantage of using the Boltzmann equation $(\mathrm{BE})$ is that large systems can be studied reasonably quickly. However, certain parameters such as phonon relaxation time $\tau(\omega)$ and phonon density of states (DOS) must be obtained either from experiments or other theoretical estimates. In addition, solving the integro-differential $\mathrm{BE}$ for general cases is nontrivial. Moreover, for nanoscale electronic devices, it is very difficult to measure directly phonon properties required to predict the thermal conductivity.

Thus, it is of great interest to develop methods for using first principles atomistic simulations to predict the thermal properties. In particular, recent advances in characterizing the interactions between atoms using classical force fields (FF) based on high level quantum mechanical calculations provide the opportunity to make first principles predictions on interesting organic and inorganic materials without additional data from experiment. This also allows the effect of microscopic structure (interfaces and surface reconstruction) to be studied quantitatively. In addition, such atomistic simulations can provide the input data (the DOS and relaxation times) to the BE approach. Thus, it can bridge between atomistic dynamics and continuum kinetics.

One can generally partition the thermal conductivity of a material into

(1) the electronic thermal conductivity, which depends on the electronic band structure, electron scattering, and electron-phonon interaction, and 
(2) the lattice conductivity, which depends mainly on the phonons (nuclear vibrations) and phonon scattering.

In this paper, we only consider the lattice thermal conductivity (as derived from the FF). This is appropriate for the systems considered here since they have large band gaps leading to small electronic contributions to thermal conduction. Because the phonon main free path in crystalline solids is much longer than that in liquids and amorphous solids, it is particularly challenging to calculate thermal conductivity for solid phase crystalline systems. Thus, to simulate the total phonon transport properties, one might expect that the periodic cell for the molecular dynamics (MD) simulations should be larger than the phonon mean free path, which can be on the order of hundreds nanometers. Moreover, calculating the phonon-phonon interactions responsible for limiting the thermal conductivity is one of the most complicated problems in solid physics. Indeed, there have been few attempts to achieve this objective (Gillan ${ }^{8}$ reviewed calculation of ionic solids by MD, Paolini ${ }^{9}$ calculated the thermal conductivity of defective ionic crystals, Li, Porter, and Yip ${ }^{10}$ studied thermal conductivity of crystalline $\beta$-SiC using atomistic simulations, Murashov ${ }^{11}$ calculated the thermal conductivity of model zeolites via MD. Recently, Volz and Chen ${ }^{12-14}$ extensively investigated thermal conductivity of silicon crystals and silicon nanowires using MD and BE).

We examine herein how to use MD simulations to provide some understanding of the various processes related to thermal conductivity and discuss the limitations in such approaches. We use the Green-Kubo (GK) relation derived from linear response theory to extract the thermal conductivity from energy current correlation functions. We find that the accuracy of thermal conductivity is sensitive to the size of the periodic unit cell in the MD simulation (which limits the phonon wavelength). However, we find that it is possible to extract an accurate thermal conductivity from periodic cells 60 times smaller than the actual phonon mean free path. The reason is that the energy current correlation time is much shorter than energy relaxation time. We illustrate this by using equilibrium MD simulations to calculate thermal conductivity of bulk crystalline diamond, including the effect on vacancies, isotopes, mass, and nanostructures. Since diamond is well known as an exceedingly good thermal conductor, these calculations on diamond crystal provide a rigorous test of our methods.

Section II outlines the theoretical background for thermal conductivity calculation. Section III provides the simulation details and the form of the FF. Section IV gives numerical results along with analysis and discussions. Finally, Sec. V summarizes the paper.

\section{THEORETICAL BACKGROUND}

\section{A. Fourier's law and the Green-Kubo relation}

The macroscopic thermal conductivity is defined from Fourier's law for heat flow under nonuniform temperature distribution. The steady state heat flow $\vec{J}_{q}$ is obtained by keeping the system and reservoirs in contact,

$$
\vec{J}_{q}=-\Lambda \cdot \nabla T
$$

where $\Lambda$ is the thermal conductivity tensor and $\vec{J}_{q}$ is the heat current produced by the temperature gradient $\nabla T$.

In energy conservation relation, the total energy current $\vec{J}_{E}$ includes both the heat current $\vec{J}_{q}$ plus the convection contribution, $\mu J$, where $\vec{J}$ is the particle current, and $\mu$ is the chemical potential. The relation between energy current and heat current is given by ${ }^{16}$

$$
\vec{J}_{q}=\vec{J}_{E}-\mu \vec{J}
$$

We will consider solids, where diffusion is negligible. In the classical limit, the energy density $h(\vec{r})$ reduces to the site energy of each particle. Therefore, heat currents can be expressed in terms of local classical properties of each particle

$$
\begin{aligned}
& \Re=\sum_{i} \vec{r}_{i} h_{i}, \\
& H=\sum_{i} h_{i}, \\
& \vec{J}_{q}=\frac{d}{d t} \Re .
\end{aligned}
$$

One approach to the simulation would be to put the system in contact with two different reservoirs with temperature $T_{1}$ and $T_{2}$. The heat current would be calculated when the system arrives at the steady state. However, with $10^{6}$ atoms in a unit cell, the system will only have dimensions of $\sim 25 \mathrm{~nm}$ on a side. Thus, even a small temperature difference of $10 \mathrm{~K}$ across this system would have a thermal gradient of $4 \times 10^{8} \mathrm{~K} / \mathrm{m}$, an unrealistically huge thermal loading for a macroscopic system. It is unlikely that linear response theory will still hold under such extreme thermal loading. Moreover, even this temperature gradient may be smaller than the thermal fluctuations in the system, making it difficult to obtain converged results in reasonable simulation times.

Instead, we use the fluctuation-dissipation theorem from linear response theory to provide the connection between the energy dissipation in irreversible processes and the thermal fluctuations in equilibrium. ${ }^{15}$ In this case, the thermal conductivity tensor can be expressed in terms of heat current correlation functions, ${ }^{15,16}$

$$
\begin{aligned}
& \Lambda=\frac{1}{k_{B} T^{2} V} \int_{0}^{\infty} d t C_{J}^{q}(t), \\
& C_{J}^{q}(t)=\left\langle\vec{J}_{q}(t) ; \vec{J}_{q}(0)\right\rangle,
\end{aligned}
$$

where $C_{J}^{q}$ is the quantum canonical correlation function, defined as ${ }^{15}$

$$
\langle a ; b\rangle=\frac{1}{\beta} \int_{0}^{\beta} d \xi \operatorname{Tr}[\rho \exp (\xi H) a \exp (-\xi H) b],
$$

where $\rho$ is the density matrix of the system at equilibrium, $\beta=1 / k_{B} T$, and $a, b$ are dynamic operators. 


\section{B. Classical limits and the semiclassical approximation}

\section{The classical limit}

Equation (7) is a quantum mechanical correlation function, which is difficult to evaluate directly in general. In this section, we discuss the classical limit of Eq. (7) and a semiclassical implementation. In the classical limit, $\hbar \rightarrow 0$, the canonical correlation function (10) reduces to the classical correlation function,

$$
C_{J}^{c}(t)=\left\langle\vec{J}_{q}(t) \vec{J}_{q}(0)\right\rangle,
$$

where $C_{J}^{c}(t)$ is obtained by phase space averaging,

$$
\langle a b\rangle=\frac{\int d \Gamma \exp (-\beta H) a b}{\int d \Gamma \exp (-\beta H)} .
$$

(Note that both classical and canonical correlation functions are even functions.) Using Eq. (9) we obtain the thermal conductivity as

$$
\Lambda^{c}=\frac{1}{k_{B} T^{2} V} \int_{0}^{\infty} d t C_{J}^{c}(t) .
$$

Usually, quantum effects are not very crucial when $T$ $\gg T_{D}$, where $T_{D}$ is the Debye temperature. Unfortunately, the Debye temperature of diamond ${ }^{17,18}$ is $\sim 1840-2000 \mathrm{~K}$, far too large to simply neglect quantum effects.

\section{Quantum corrections}

To investigate the quantum corrections, we first introduce the one-side quantum correlation function of heat current, $C_{J}^{+}(t)$ and $C_{J}^{-}(t)$,

$$
\begin{aligned}
& C_{J}^{+}(t)=\operatorname{Tr}\left[\rho \vec{J}_{q}(t) \vec{J}_{q}(0)\right], \\
& C_{J}^{-}(t)=\operatorname{Tr}\left[\rho \vec{J}_{q}(0) \vec{J}_{q}(t)\right] .
\end{aligned}
$$

It can be proved ${ }^{19}$ that $C_{J}^{+}$and $C_{J}^{-}$satisfy

$$
C_{J}^{+}(t)=\left[C_{J}^{-}(t)\right]^{*} .
$$

Using Eq. (14) in Eq. (7) leads to

$$
C_{J}^{q}(t)=\frac{1}{\beta} \int_{0}^{\beta} d \xi C_{J}^{+}(t+i \xi \hbar) .
$$

In order to estimate the quantum correction to the classical equation, Eq. (11), we need to find a relationship between the quantum canonical correlation function and its classical counterpart. Several authors have suggested methods that approximately map classical correlation functions to quantum ones. Schofield ${ }^{20,21}$ suggested

$$
C^{+}(t)=C^{c}\left(t-\frac{i \hbar \beta}{2}\right),
$$

which satisfies the detailed balance requirement.

Using Eq. (16), we can expand the right-hand side into power series of $\hbar$,

$$
C^{q+}(t)=C^{c}(t)-\frac{i \hbar \beta}{2} \dot{C}^{c}(t)-\frac{\hbar^{2} \beta^{2}}{8} \ddot{C}^{c}(t)+o\left(\hbar^{3}\right) .
$$

Substituting Eq. (17) into Eq. (6), leads to

$$
\boldsymbol{\Lambda}=\boldsymbol{\Lambda}_{\mathbf{c}}+\frac{\hbar^{2} \beta^{2}}{24 k_{B} T^{2} V} \dot{C}^{c}(0)+o\left(\hbar^{4}\right),
$$

where $\boldsymbol{\Lambda}_{\mathbf{c}}$ is obtained from Eq. (11). However, due to the time reversal symmetry of the classical correlation function, the second term is zero. Similarly, the higher order terms are zero. Therefore, if Eq. (16) is adopted, the quantum thermal conductivity is the same as the classical counterpart.

Egelstaff $^{22}$ also suggested a somewhat similar but more complex approximation. In addition, there have been some attempts to calculate quantum correlation functions from path integral and semiclassical approaches. The approximation by Egelstaff is

$$
C^{+}(t)=C^{c}\left(\sqrt{t^{2}-i \hbar \beta t}\right),
$$

which leads to a slightly different relationship between the classical and quantum descriptions of thermal conductivity. Rather than Eq. (18), we obtain

$$
\boldsymbol{\Lambda}=\boldsymbol{\Lambda}_{\mathbf{c}}+\frac{\hbar^{2} \beta^{2}}{12 k_{B} T^{2} V} \int_{0}^{\infty} d t \frac{\dot{C}^{c}(t)}{t}+o\left(\hbar^{4}\right) .
$$

The second term on the right-hand side appears to be a correction to the classical thermal conductivity. However, our simulation results (Sec. IV) indicate that the correction term for diamond in Eq. (20) is six orders of magnitude smaller than the leading term $\boldsymbol{\Lambda}_{\mathbf{c}}$.

We should note that neither Eq. (16) nor Eq. (19) is exact. Thus, it is reasonable that they lead to slightly different answers. However, we will see that the difference between them is negligible, giving us confidence in the reliability of the classical description. In Sec. II B 3, we will take a closer look at the mapping relations from the perspective of harmonic analysis.

\section{Harmonic analysis}

Here, we employ a harmonic analysis to examine the quantum correlations. First, we partition the quantum correlation function into the real and imaginary parts,

$$
C^{+}(t)=C^{\prime}(t)+i C^{\prime \prime}(t),
$$

with

$$
\begin{aligned}
& C^{\prime}(t)=\frac{1}{2}[\langle O(t) O(0)\rangle+\langle O(0) O(t)\rangle], \\
& C^{\prime \prime}(t)=-\frac{i}{2}[\langle O(t) O(0)\rangle-\langle O(0) O(t)\rangle] .
\end{aligned}
$$

where $O$ is a dynamic operator, and \langle\rangle denotes the canonical averaging. $C^{\prime}(t)$ is an even real function, while $C^{\prime \prime}(t)$ is an odd real function.

To relate the canonical correlation function to the one side quantum correlation function, we use the Fourier transform of Eq. (15),

$$
\widetilde{C}_{J}^{q}(\omega)=\frac{1}{\beta \hbar \omega}[\exp (\beta \hbar \omega)-1] \widetilde{C}_{J}^{+}(\omega) .
$$


From the detailed balance relation ${ }^{19}, \widetilde{C}^{+}(\omega)$ $=\exp (-\beta \hbar \omega) \widetilde{C}^{-}(\omega)$, we can express Eq. (24) in terms of the real function $\widetilde{C}^{\prime}(\omega)$,

$$
\widetilde{C}_{J}^{q}(\omega)=\frac{2}{\beta \hbar \omega} \tanh \frac{\beta \hbar \omega}{2} \widetilde{C}^{\prime}(\omega) .
$$

To further investigate the relationship between quantum and classical thermal conductivity, we generalize the definition of the static thermal conductivity by introducing the frequency-dependent thermal conductivity, $\Lambda(\omega)$,

$$
\begin{aligned}
\Lambda(\omega) & =\frac{1}{2 k_{B} T^{2} V} \int_{-\infty}^{\infty} C_{J}^{q}(t) e^{i \omega t} d t \\
& =\frac{1}{2 k_{B} T^{2} V} \widetilde{C}_{J}^{q}(\omega) .
\end{aligned}
$$

Substituting Eq. (24) into Eq. (26), leads to

$$
\Lambda(\omega)=\frac{1}{2 k_{B} T^{2} V} \frac{2}{\beta \hbar \omega} \tanh \frac{\beta \hbar \omega}{2} \widetilde{C}^{\prime}(\omega) .
$$

Up to this point, no approximation to the correlation functions have been made. All these equations are exact.

\section{Harmonic limits}

For harmonic systems bilinearly coupled to a harmonic reservoir, the real part of the quantum correlation function has a simple relation with its classical counterpart in the frequency space, ${ }^{23}$

$$
\widetilde{C}^{\prime}(\omega)=\frac{1}{2}\left[\widetilde{C}^{+}(\omega)+\widetilde{C}^{-}(\omega)\right]=\frac{\beta \hbar \omega}{2} \operatorname{coth} \frac{\beta \hbar \omega}{2} \widetilde{C}^{c}(\omega) .
$$

Similarly, the imaginary part connects with the classical correlation function via

$$
\widetilde{C}^{\prime \prime}(\omega)=\frac{\beta \hbar \omega}{2} \widetilde{C}^{c}(\omega)
$$

Using Eq. (28) in Eq. (27), leads to

$$
\Lambda(\omega)=\frac{1}{2 k_{B} T^{2} V} \widetilde{C}_{J}^{c}(\omega) .
$$

This leads to the classical thermal conductivity as

$$
\boldsymbol{\Lambda}(0)=\boldsymbol{\Lambda}_{c},
$$

which is exactly Eq. (18).

This result can be understood as follows: Equation (28) is only exact for harmonic oscillators. For a purely harmonic system each normal mode is uncoupled with others. In other words, phonon modes do not interact with one another. Therefore, the only contribution to the thermal resistance for a macroscopic harmonic system is from phonon modes with wavelengths comparable to or longer than the macroscopic length scale (where boundary scattering can couple these modes). This leads to the zero frequency mode condition in Eq. (26). Since no $\hbar$ is involved in Eq. (30), this result is exact for harmonic systems. Therefore, the pure classical results are exactly the same as quantum results for harmonic systems; no quantum correction is needed.
A similar result is obtained for vibrational relaxation, ${ }^{23}$ where both the full quantum relaxation rate and full classical relaxation rate are the same for harmonic systems. Fundamentally, the finite thermal conductivity arises from the coupling strength among system vibrational modes. The processes involved in such a harmonic system are related to those in vibrational relaxation. This explains the underlying reason for the higher order terms in Eq. (18) to be zero.

Unfortunately, real systems are not exactly harmonic. The higher order terms in the Taylor expansion of the potential surface will contribute to the coupling of phonon modes. These anharmonicities are essential to explain in many physical phenomena in solids, including thermal conductivity. Because of anharmonicity, phonons can be created, destroyed, or scattered, leading to a finite phonon mean free path and finite thermal conductivity. The magnitude of these anharmonic effects depends on the material properties (the interactions and structure).

Since an accurate FF will generally account for anharmonic effects, we may use MD to study thermal conductivity. Such MD simulations implicitly include the vibrational mode couplings. Many processes involved in thermal conductivity such as boundary scattering, crystal imperfections, and isotope effects can all be included in MD simulations, although they are modeled classically. Equation (27) suggests that we now need only to find a method to map the classical correlation functions into the quantum counterparts. Although Eq. (28) is only exact for harmonic systems, we can still use it to approximate the quantum correlation. Since the classical counterpart already includes anharmonic behavior, the approximate quantum correlation function will also have anharmonicity partially included.

Cao and Voth ${ }^{24}$ suggested that replacing $\widetilde{C}^{c}(\omega)$ in Eq. (28) by a classical correlation function calculated from centroid potential surface provides a much better approximation for the quantum correlation function. The details on how to obtain the centroid potential surface are beyond the scope of this paper. Nevertheless, we can still estimate roughly the first-order quantum correction by considering the leading quantum correction term to the pure classical potential, ${ }^{25}$

$$
\begin{aligned}
& V_{q}\left(\left\{r_{i}\right\}\right)=V\left(\left\{r_{i}\right\}\right)+\Delta V\left(\left\{r_{i}\right\}\right), \\
& \Delta V\left(\left\{r_{i}\right\}\right)=\frac{\hbar^{2} \beta}{24} \sum_{i} \frac{1}{m_{i}} \frac{\partial^{2} V}{\partial^{2} r_{i}},
\end{aligned}
$$

where $V_{q}$ is the quantum modified potential surface, and $\left\{r_{i}\right\}, i=1, \ldots, 3 N$ denotes the particle coordinates. The second term in Eq. (32) modifies the interactions in the MD simulation to partially include some quantum effects. It is obvious that the new potential has a less shallow well and a lower barrier than the original classical potential. This can be understood as partially accounting for zero point energy and tunneling effects. Of course, at high temperatures the modified potential becomes the same as the classical potential. To estimate the correction to the classical correlation functions from the modified potential, we can expand the interaction potential in Eq. (32) to the first order in the density matrix, 


$$
\begin{aligned}
C_{q}^{c}(t)= & \operatorname{Tr}\left[\exp (-\beta H-\beta \Delta V) \vec{J}_{q}(t) \vec{J}_{q}(0)\right] / \\
& \operatorname{Tr}[\exp (-\beta H-\beta \Delta V)] \\
= & C_{J}^{c}(t)-\beta\left[\left\langle\Delta V \vec{J}_{q}(t) \vec{J}_{q}(0)\right\rangle_{c}\right. \\
& \left.-C_{J}^{c}(t)\langle\Delta V\rangle_{c}\right]+o\left[(\beta \Delta V)^{2}\right],
\end{aligned}
$$

where \langle\rangle$_{c}$ indicates phase space averaging over the classical density matrix, and $C_{q}^{c}(t)$ is the classical correlation function on the modified potential with the first-order quantum correction. Replacing $\widetilde{C}_{J}^{c}(\omega)$ in Eq. (30) by the Fourier transform of $C_{q}^{c}(t)$, we then have the first-order quantum correction,

$$
\begin{aligned}
\boldsymbol{\Lambda}(0) \approx & \boldsymbol{\Lambda}_{c}-\beta\left[\frac{1}{k_{B} T^{2} V} \int_{0}^{\infty} d t\left\langle\Delta V \vec{J}_{q}(t) \vec{J}_{q}(0)\right\rangle_{c}\right. \\
& \left.-\boldsymbol{\Lambda}_{c}\langle\Delta V\rangle_{c}\right] .
\end{aligned}
$$

In a harmonic system, $\Delta V$ is constant for each vibrational mode in the normal mode space. In this case, the second term in Eq. (34) and all the higher order terms vanish, as expected.

In this section, we have discussed the relationship between the classical lattice thermal conductivity and its quantum counterpart. The corrections to the classical thermal conductivity due to quantum effects are discussed based on the quantum corrections to the fundamental heat correlation functions. This provides insight for the role that quantum effects play in lattice thermal conductivity, and it also shows the fundamental reason why classical simulations can often capture correct thermal conductivities in many cases. In addition to the quantum corrections made to the fundamental quantities (i.e., heat correlation functions), another simpler but less rigorous temperature scaling method has been used to qualitatively include quantum effects, ${ }^{12,14}$

$$
3 N k_{B} T_{\mathrm{MD}}=\int_{0}^{\omega_{D}} d \omega D(\omega) n(\omega, T) \hbar \omega .
$$

In order to use the above-mentioned approximation, however, extra calculations have to be performed to obtain the phonon density of states, $D(\omega)$, and the Debye frequency, $\omega_{D}$. The low frequency density of states, responsible for heat transport, are not trivial to calculate accurately. More important, Eq. (35) tends to overestimate $T_{\mathrm{MD}}$ because of the contribution from high frequencies in the spectrum that are insignificant for heat transport. In other words, the phonon modes that are most important for thermal conduction are those with very low frequencies [i.e., Eq. (25)], and these modes are nearly classical. Therefore, we argue that Eq. (35) usually is not necessary for thermal conductivity calculations, although it is important for calculating heat capacity where high frequency modes contribute significantly. ${ }^{10}$ This conclusion is consistent with Eqs. (18), (20), (31), and (34). In fact, the correct quantum heat capacity can also be derived from Eq. (28) through velocity correlation functions.

\section{SIMULATION DETAILS}

\section{A. The force field}

For the illustrative calculations in this paper, we use the Brenner bond-order-dependent FF. This FF can be written formally as summation of pairwise interactions, ${ }^{26}$

$$
\begin{aligned}
V_{\mathrm{tot}} & =\sum_{i} \sum_{j>i}\left[V_{R}\left(r_{i j}\right)-\bar{B}_{i j} V_{A}\left(r_{i j}\right)\right], \\
& =\sum_{i} \sum_{j>i} V_{i j},
\end{aligned}
$$

where $V_{R}$ and $V_{A}$ are the repulsive and attractive part of the pairwise binding potential, respectively. They have the same form as a general Morse potential, ${ }^{26}$

$$
\begin{aligned}
& V_{R}\left(r_{i j}\right)=f_{i j}\left(r_{i j}\right) \frac{D_{i j}^{e} S_{i j}}{S_{i j}-1} \exp \left[-\sqrt{2 S_{i j}} \beta_{i j}\left(r_{i j}-R_{i j}^{e}\right)\right], \\
& V_{A}\left(r_{i j}\right)=f_{i j}\left(r_{i j}\right) \frac{D_{i j}^{e} S_{i j}}{S_{i j}-1} \exp \left[-\sqrt{\frac{2}{S_{i j}}} \beta_{i j}\left(r_{i j}-R_{i j}^{e}\right)\right],
\end{aligned}
$$

where

$$
f_{i j}(r)=\left\{\begin{array}{cc}
1, & r \leqslant R_{i j}^{(1)} \\
\left\{1+\cos \left[\frac{\pi\left(r-R_{i j}^{(1)}\right)}{R_{i j}^{(2)}-R_{i j}^{(1)}}\right]\right\} / 2, & R_{i j}^{(1)}<r<R_{i j}^{(2)} \\
0, & r \geqslant R_{i j}^{(2)} .
\end{array}\right.
$$

$f_{i j}(r)$ is a cutoff function that explicitly restricts the interaction within the nearest neighbors. The bond order parameter $\bar{B}_{i j}$ depends on the environment around atoms $i$ and $j$ and is written as ${ }^{26}$

$$
\bar{B}_{i j}=\frac{B_{i j}+B_{j i}}{2}+F_{i j}\left(N_{i}^{H}(t), N_{j}^{C}(t), N_{i j}^{\mathrm{conj}}\right),
$$

In Eq. (40), $B_{i j}$ implicitly contains many-body information. The detailed formulation and all parameters are given by Brenner. ${ }^{26}$

\section{B. The heat flux}

As indicated in Eq. (36), the interaction potential is formally pairwise, allowing the site energy $h_{i}$ in Eq. (3) to be defined as

$$
h_{i}=\frac{p_{i}^{2}}{2 m_{i}}+\frac{1}{2} \sum_{j} V_{i j} .
$$

Therefore, the heat current can be calculated through Eq. (5). Because $V_{i j}$ is in fact a many-body potential, ${ }^{20}$ the calculation of heat current $\vec{J}_{q}(t)$ is much more complicated than for a truly pairwise FF. After some algebra steps, $\vec{J}_{q}$ is given by

$$
\vec{J}_{q}(t)=\sum_{i} \vec{v}_{i} h_{i}+\frac{1}{2} \sum_{i, j} \sum_{k, l} \vec{r}_{i k} \vec{F}_{i j}^{k l} \cdot \vec{v}_{i},
$$

with 


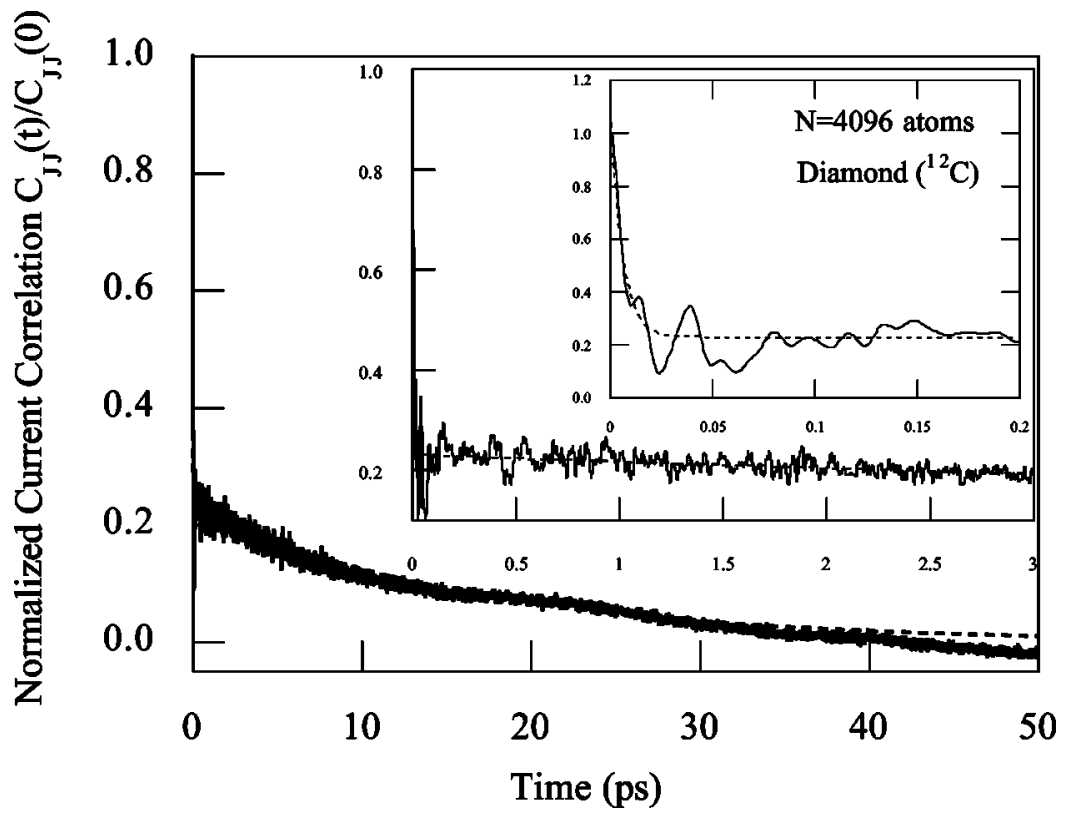

FIG. 1. The heat current correlation function and the double exponential fitting for the $N=4096$ atom simulation of ${ }^{12} \mathrm{C}$ diamond $\left(T=300 \mathrm{~K}, t_{\text {run }}=400 \mathrm{ps}\right.$. The insets show the short time region at different scales. The parameters for the fitted double exponential function (dashed lines) are given in Table I.

$$
\vec{F}_{i j}^{k l}=-\frac{\partial V_{k l}}{\partial \vec{r}_{i j}} .
$$

In deriving Eqs. (42) and (43), we implicitly imposed the condition that there is no net momentum for the system. For a truly pairwise FF, Eq. (42) recasts to the familiar form ${ }^{15,27}$

$$
\vec{J}_{q}(t)=\sum_{i} \vec{v}_{i} h_{i}+\frac{1}{2} \sum_{i, j} \vec{r}_{i j} \vec{F}_{i j} \cdot \vec{v}_{i} .
$$

\section{Dynamic systems}

A 1 fs time step was employed in all MD simulations. For each system, we initially equilibrated the system using Gaussian thermostat MD $(300 \mathrm{~K})$ for $40 \mathrm{ps}$. After equilibration, we carried out 400 ps of constant energy ( $N V E$ ) MD, calculating the heat current for every time step.

All simulations on diamond started with a cubic unit cell constant of $3.556 \AA$. This unit cell was extended to build large supercells for the MD simulations (thus, an $8 \times 8 \times 8$ cell leads to 4096 atoms). Periodic boundary conditions were applied to the super cell in all three directions.

\section{NUMERICAL RESULTS AND ANALYSIS}

\section{A. Bulk diamond, isotopically pure}

To test the convergence of MD simulations on thermal conductivity, we carried out simulations for cubic diamond, described with supercells containing $512(4 \times 4 \times 4), 1000$ $(5 \times 5 \times 5), \quad 1728(6 \times 6 \times 6), 2744(7 \times 7 \times 7), 4096(8$ $\times 8 \times 8)$, and $8000(10 \times 10 \times 10)$ atoms. Since diamond is isotopic, we used the scalar thermal conductivity that is the average value of three principle directions (which reduces the uncertainty in the theoretical values). As discussed in previous sections, the phonon mean free path is the limiting factor to obtaining accurate results. For too small a simulation cell, the time for phonon to travel through the simulation cell is much shorter than the decay time of the current correlation function. This causes the phonons to be scattered more frequently than they would be in the infinite system. In this case, only the short time correlation function is accurate.

Even so we can estimate the thermal conductivity from simulations using periodic cells smaller than the mean free path. Thus, based on the macroscopic law of relaxation and Onsager's postulate for microscopic thermal fluctuation, we expect that the asymptotic decay of the heat correlation function will be exponential. Since the kinetic coefficients depend mostly on long time decay rate behavior and since an exponential-decay behavior is exhibited in a microscopic time scale, we expect that a medium-sized simulation cell can be used to extract the decay rate of the heat dissipation.

Figure 1 shows the heat current correlation functions at short times for a cubic diamond crystal with 4096 atoms. We see a rapid initial decay $(\sim 50 \mathrm{fs}$ ) followed by a long time exponential decay of the correlation function. Probably the initial fast decay is due to high frequency optical modes in the crystal, which do not significantly contribute to thermal conductivities (either conceptually and computationally), because they couple only weakly to the low frequency acoustic modes and they are little populated at room temperature.

To fit the correlation function we used a double exponential function

$$
C_{J}^{c}(t)=A_{o} \exp \left(-t / \tau_{o}\right)+A_{a} \exp \left(-t / \tau_{a}\right), \quad t \geqslant 0,
$$

where the subscripts $o$ and $a$ denote fast optical modes and slow acoustic modes, respectively. The thermal conductivity is then given by

$$
\lambda=\frac{1}{k_{B} T^{2} V}\left(A_{o} \tau_{o}+A_{a} \tau_{a}\right) .
$$

The parameters $A_{o}, \tau_{o}, A_{a}$, and $\tau_{a}$, are derived from the first 3 ps using nonlinear least-squares methods (MazquazltLevenberg). This leads to the results in Table I, where we see that high frequency optical modes contribute only $0.1 \%$ to the thermal conductivity. Note that the current correlation function at very short time ( $\leqslant 20 \mathrm{fs}$ ) is not exponential in the 
TABLE I. Parameters for the double exponential fitting of the heat current correlation functions for ${ }^{12} \mathrm{C}$ diamond based on Eq. (45). $A_{o} / V$ $=810.5(\mathrm{kcal} / \mathrm{mol})^{2} /\left(\mathrm{Aps}^{2}\right)$ and $A_{a} / V=189.6(\mathrm{kcal} / \mathrm{mol})^{2} /\left(\AA \mathrm{ps}^{2}\right)$.

\begin{tabular}{ccccl}
\hline \hline Atoms & $A_{o} / V$ & $\tau_{o}(\mathrm{ps})$ & $A_{a} / V$ & $\tau_{a}(\mathrm{ps})$ \\
\hline 512 & 822.0 & 0.00575 & 138.9 & 10.605 \\
1000 & 805.3 & 0.00592 & 147.2 & 10.608 \\
1728 & 813.6 & 0.00577 & 198.0 & 16.393 \\
2744 & 814.7 & 0.00575 & 181.8 & 14.719 \\
4096 & 811.0 & 0.00571 & 233.9 & 16.527 \\
8000 & 808.1 & 0.00582 & 176.3 & 16.656 \\
$\infty$ & 810.5 & 0.0058 & 189.6 & 16.66 \\
\hline \hline
\end{tabular}

strict sense, since only Markovian processes have an exponential decay correlation function. In most physical processes, nonexponential decay of the correlation function near $t=0$ is quite common. However, for the cases studied here, this does not make a significant difference in the final calculated thermal conductivity. Therefore, it is satisfactory to use an exponential function to fit the initial trend. (This is the cause of the fitting function overshooting the value at the origin.)

Figure 2 shows the dependence of the calculated thermal conductivities on the size of the simulation cell. The uncertainties shown here are estimated by $\sqrt{2 \tau / t_{\text {run }}}$ where $t_{\text {run }}$ is the simulation length. When the simulation cell is too small, the particles in the simulation cell do not have sufficient time to lose their previous dynamic information before a periodically equivalent phonon travels to the same place. As a result the longer correlation functions $\left(\tau_{a}\right)$ are contaminated from memory effects. Thus, for very small simulation cells, only the very short time correlation function resembles the real system behavior. This is shown in Table I where $\tau_{a}$ is independent of size. From Fig. 2 and Table I, we see that a cell of $\sim 28.44 \AA \quad(N=4096$ atoms $)$ is required to obtain a converged thermal conductivity.

In kinetic theory, the thermal conductivity is given by, ${ }^{28}$

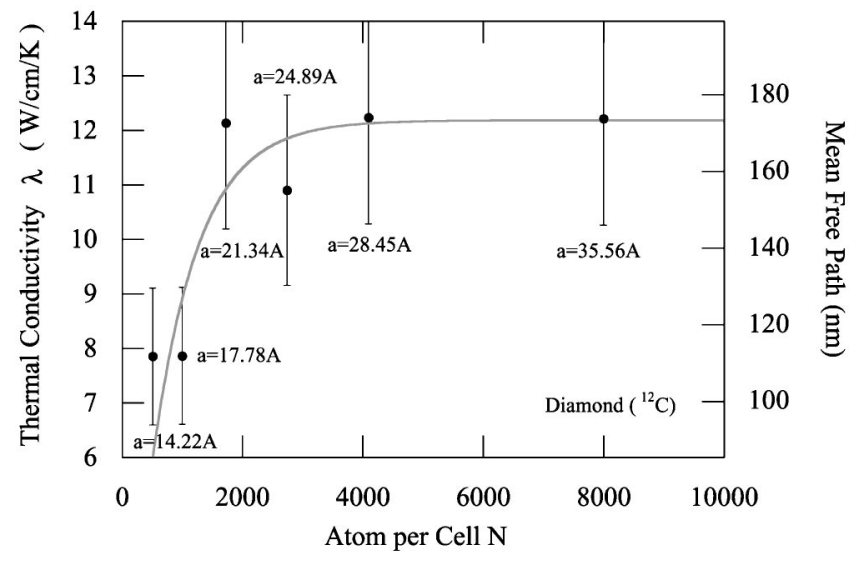

FIG. 2. The thermal conductivity of isotopically pure ${ }^{12} \mathrm{C}$ diamond as a function of simulation cell size. The size of cubic diamond supercells are indicated. The error bar is estimated from $\left(2 \tau_{o} / t_{\text {run }}\right)^{1 / 2}$ with $t_{\text {run }}=400 \mathrm{ps}$. The right $Y$ coordinate indicates the length of average phonon mean free path $L=3 \lambda / C_{v} v$ where $C_{v}$ (the constant volume heat capacity) is calculated from quantum corrected velocity correlation function (Ref. 29), which gives $C_{v}=0.12 \mathrm{cal} / \mathrm{g} / \mathrm{K}$ and the velocity of sound, $v$, is obtained from $v$ $=1 / \sqrt{\rho \beta_{T}}=12 \mathrm{~km} / \mathrm{s}$.

$$
\lambda \approx \frac{1}{3} C v L,
$$

where $C$ is the specific heat, and $v$ is the speed of sound, and $L$ is the phonon mean free path. Our simulations (based on the Brenner FF) lead to $\rho=3.5 \mathrm{~g} / \mathrm{cm}^{3}, C_{v}=0.12 \mathrm{cal} / \mathrm{g} / \mathrm{K}$ (calculated from quantum corrected velocity correlation functions ${ }^{29}$ ), a compressibility of $\beta_{T}=0.0020 \mathrm{GPa}^{-1}$ and hence a speed of sound of $v=1 / \sqrt{\rho \beta_{T}}=12.0 \mathrm{~km} / \mathrm{s}$. Using $\lambda$ $=12.2 \mathrm{w} / \mathrm{cm} / \mathrm{K}$, Eq. (47) leads to a mean free path for acoustic phonons of $L=174 \mathrm{~nm}$. The experimental values ${ }^{30,31}$ are $\rho=3.5 \mathrm{~g} / \mathrm{cm}^{3}, C_{p}=0.11 \mathrm{cal} / \mathrm{g} / \mathrm{K}, \beta_{T}=0.0022 \mathrm{GPa}^{-1}$, and $v$ $=11.4 \mathrm{~km} / \mathrm{s}$. Using the experimental value of $\lambda$ $=33 \mathrm{w} / \mathrm{cm} / \mathrm{K}$, leads to $L=494 \mathrm{~nm}$.

The comparison of the values for $\rho, C_{v}, \beta_{T}$, and $v$ from theory using the Brenner potential are within $10 \%$ of experimental values. Thus it is surprising that the calculated $\lambda$ is $60 \%$ smaller than the experimental results. As discussed in Sec. IV B 1, the calculated thermal expansion is 3.4 times higher than experimental value, suggesting that the Brenner potential is too anharmonic. This will lead to much smaller values for $\lambda$, as observed.

Although the phonon mean free path $L \sim 1740 \AA$ is much larger than the cell length, we still obtain a converged value for $\lambda$ of $a=28.44 \AA$ This is because we need not seek a completely converged correlation function, which would require a cell of $N=5.9 \times 10^{8}$ atoms. Instead, we need only to extract the $\tau_{a}$ decay constant from the initial correlation function. Table I lists the exponential decay constants of the correlation functions for systems of different sizes. The weighting factors $A_{o}$ and $A_{a}$ are normalized by the volume and the values for various $N$ are averaged over all $N$ to reduce the error. The fitted relaxation times are $\tau_{o}$ $=0.0058 \mathrm{ps}$ and $\tau_{a}=16.6 \mathrm{ps}$.

\section{B. Defects}

The above-mentioned calculations assumed a perfect crystal, where the thermal scattering is due solely to anharmonic vibrations of the atoms. However, real materials have many defects. We consider here the effect of isotopic variations, vacancies, and mass variations for composite systems.

\section{Isotopic substitutions}

The natural abundance of ${ }^{13} \mathrm{C}$ is $1.1 \%$. Since the phonon frequency depends on mass this leads to local fluctuations in the natural frequency which can lead to increased phonon scattering. Thus, natural diamond crystals always contain a significant number of scattering centers. The decreased phonon mean free path due to increased scattering makes it easier to calculate $\lambda$ since the correlation function convergences faster, allowing a smaller supercell to be used in the MD simulations.

Indeed, experiments by Anthony et al. ${ }^{31}$ found that the thermal conductivity of diamond at room temperature increased by $50 \%$ when the concentration of isotope ${ }^{13} \mathrm{C}$ was reduced by a factor of 15 ! Their remarkable discovery led to a patent ${ }^{32}$ and stimulated a number of studies to understand the sensitive behavior of diamond thermal conductivity to the isotopic purity. Theoretical investigations and further experiments concluded the main effect of isotopic substitution 

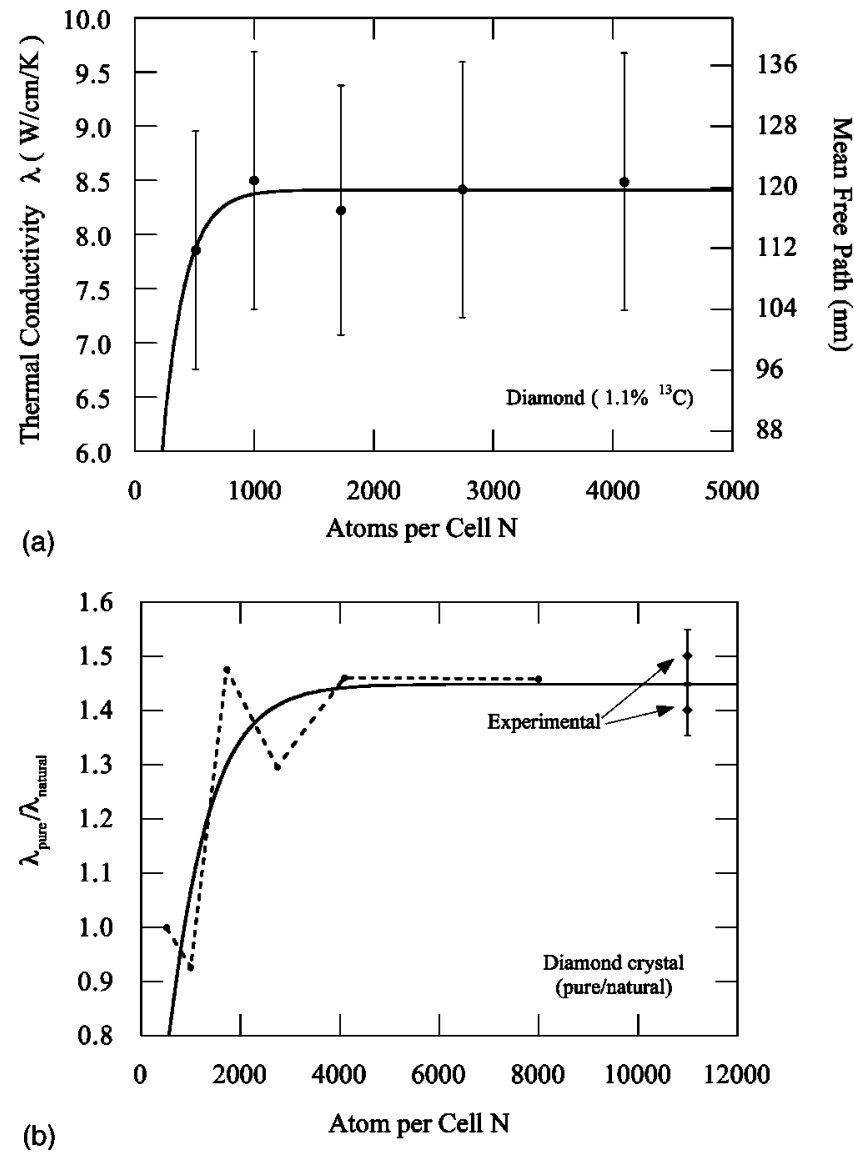

FIG. 3. (a) The thermal conductivities for the perfect diamond structure with $1.1 \%$ natural abundance of ${ }^{13} \mathrm{C}(T=300 \mathrm{~K})$. The right $Y$ coordinate indicates the average phonon mean free path. The error bar is estimated from $\left(2 \tau / t_{\text {run }}\right)^{1 / 2}$ with $t_{\text {run }}=400 \mathrm{ps}$. (b) Ratio of thermal conductivity for pure ${ }^{12} \mathrm{C}$ diamond with respect to that for $1.1 \%{ }^{12} \mathrm{C}$. The experimental values are from references by Anthony (Ref. 31) and Olson (Ref. 35).

on the thermal conductivity is due to normal $(N)$ processes of the phonon scattering in diamond crystals, ${ }^{17,33-35}$ which conserves the total wave vector of scattering phonons. The Umklapp $(U)$ processes of phonon scattering, in which the total wave vector changes by a reciprocal latter vector, also contribute. Our MD simulations contain no presumptions about the importance of $U$ process vs $N$ processes. Indeed, we were concerned whether a purely classical calculation would capture the substance of isotopic scattering. In principle, the classical dynamics should include such contributions, and hence, an important and effective test of our classical methods is to verify the remarkable isotope effect on diamond thermal conductivity.

Figure 3(a) shows the calculated thermal conductivities of diamond containing the $1.1 \%$ natural abundance of ${ }^{13} \mathrm{C}$. We chose the sites randomly. The results are clear from Fig.
3. First, the calculated thermal conductivity for diamond with natural abundance ${ }^{13} \mathrm{C}$ is much lower than for pure ${ }^{12} \mathrm{C}$. Indeed, we calculate that

$$
\frac{\lambda\left({ }^{12} \mathrm{C}\right)}{\lambda(\text { natural abundance })}=1.45 \pm 0.16
$$

which compares well with the experimental values ${ }^{31,35}$ of $1.50 \pm 0.05$ and 1.4. The ratio of increasing thermal conductivity in pure diamond is depicted in Fig. 3(b). This result confirms the conclusion ${ }^{17,33-35}$ that no additional defects are necessary to explain the large isotope effect in natural diamond. Thus, the MD calculations explain the surprisingly high sensitivity to isotopic substitution.

Comparing Figs. 3(a) and 2, it is clear that including isotopic variations leads to much faster convergence with the simulation cell in the calculation of thermal conductivity than the pure ${ }^{12} \mathrm{C}$ crystal. This is due to the decreased phonon mean free path.

We used the Brenner FF in our calculations. This FF was not optimized for describing the crystal properties of diamond important for phonons (e.g., elastic constants, thermal expansion constant, phonon dispersion relations, etc.). It leads to (i) a compressibility of $0.0020 \mathrm{GPa}^{-1}$ compared to the experimental value of $0.0022 \mathrm{GPa}^{-1}$ and (ii) a phonon frequency ${ }^{36}$ at the $\Gamma$ point of $1288 \mathrm{~cm}^{-1}$ compared with the experimental value of $1333.9 \mathrm{~cm}^{-1}$. These calculated properties (including heat capacity) agree reasonably well with experimental values. This is not surprising since the Brenner FF parameters were optimized to fit properties at low temperature. However, this FF fails to accurately describe the diamond crystal anharmonicity vital to the thermal conductivity. Thus as shown in Sec. IV A, the Brenner FF predicts the linear thermal expansion coefficient at room temperature $^{37}$ (see Table II) to be $\beta=4.16 \times 10^{-6} \mathrm{~K}^{-1}$, much larger than experimental value ${ }^{38} \beta=1.18 \times 10^{-6} \mathrm{~K}^{-1}$. This indicates that the Brenner FF is too anharmonic. Since the thermal conductivity decreases dramatically with increasing thermal expansion coefficient, ${ }^{39}$ we believe that the large anharmonicity of the Brenner potential is responsible for the calculated thermal conductivity for natural diamond $(\lambda$ $=8.4 \mathrm{w} / \mathrm{cm} / \mathrm{K}$ at $300 \mathrm{~K}$ ) being significantly lower than the experimental value, $\lambda=21.9 \mathrm{w} / \mathrm{cm} / \mathrm{K}$ at $298 \mathrm{~K}$.

We expect that a FF with a more accurate description of the anharmonicity would lead to better agreement with the experimental magnitude for the thermal conductivity of diamond. Thus, our earlier calculations ${ }^{40}$ on $\mathrm{C}_{60}$ crystals, which used a very accurate $\mathrm{FF}$, led to $\lambda=4 \times 10^{-3} \mathrm{~W} / \mathrm{cm} / \mathrm{K}$, in good agreement with the experimental value of $\lambda$ $=0.4 \mathrm{~W} / \mathrm{m} / \mathrm{K}$. Although the calculated thermal conductivity $\lambda$ for diamond differs substantially from experiment, we ex-

TABLE II. The volumes of simulation cells of 2744 atoms at various temperatures. The experimental lattice constant at $298 \mathrm{~K}$ is $3.56688 \AA$, which gives the volume of the cell $15565.4 \AA^{3}$.

\begin{tabular}{ccccccc}
\hline \hline Temperature $(\mathrm{K})$ & 0 & 247.7 & 297.3 & 346.8 & 396.2 & 495.1 \\
\hline Volume $\left(\AA^{3}\right)$ & 15446.2 & 15494.8 & 15505.6 & 15515.1 & 15524.5 & 15543.0 \\
\hline \hline
\end{tabular}




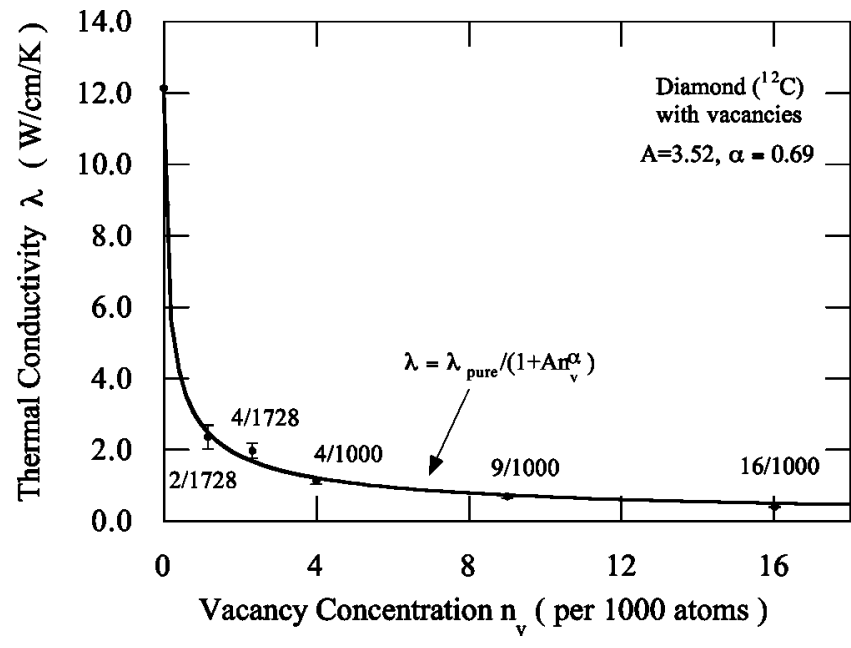

FIG. 4. The thermal conductivities of ${ }^{12} \mathrm{C}$ diamond containing random distributions of vacancies. The solid curve is the fitting to the function Eq. (51), where $\alpha=0.69$, and $A=3.52$, see details in the text.

pect that relative changes in the thermal conductivity due to defects are valid. This view is supported by the accurate prediction of the isotope effect.

\section{Vacancies}

As a second application, we studied the effects of vacancies on the thermal conductivity. Here we considered a cell with $N=1000$ or 1728 atoms and included a random distribution of vacancies, leading to the results in Fig. 4, which shows that the thermal conductivity decreases rapidly with vacancy concentration, $n_{v}$. Assuming that vacancy scattering is independent of the acoustic scattering dominant in the pure perfect crystal, we expect the total phonon scattering length $(L)$ to be

$$
L_{\text {tot }}^{-1} \approx L_{\text {pure }}^{-1}+L_{\text {vac }}^{-1},
$$

where $L_{\text {pure }}$ denotes the scattering length in perfect crystal and $L_{\mathrm{vac}}$ denotes the scattering length induced by vacancies. Assuming that the sound velocity and heat capacity are not affected by the vacancies, Eq. (49) leads to

$$
\lambda_{\text {tot }}\left(n_{v}\right)=\frac{\lambda_{\text {pure }} \lambda_{\text {vac }}}{\lambda_{\text {pure }}+\lambda_{\text {vac }}}=\frac{\lambda_{\text {pure }}}{1+\lambda_{\text {pure }} / \lambda_{\text {vac }}} .
$$

Figure 4 shows the results for $n_{v}$ up to $1.6 \%$ which are accurately fitted by Eq. (50), with

$$
\lambda_{\text {tot }}\left(n_{v}\right)=\frac{\lambda_{\text {pure }}}{1+A n_{v}^{\alpha}},
$$

where $A=413.6( \pm 10.8 \%)$ and $\alpha=0.69( \pm 0.11)$.

The fit in Eq. (51) suggests that the vacancy contribution to thermal conductivity leads to a scaling law of

$$
\lambda_{\mathrm{vac}} \propto n_{v}^{-\alpha},
$$

where $\alpha \sim 0.7$. This result agrees with the phenomenological theory, ${ }^{7}$ which estimates the exponent to be $1 / 2$ to $3 / 4$.

Our calculations indicate that with vacancies (as for the pure systems) the fast decay in the heat current correlation function integrates to a very small contribution to the overall

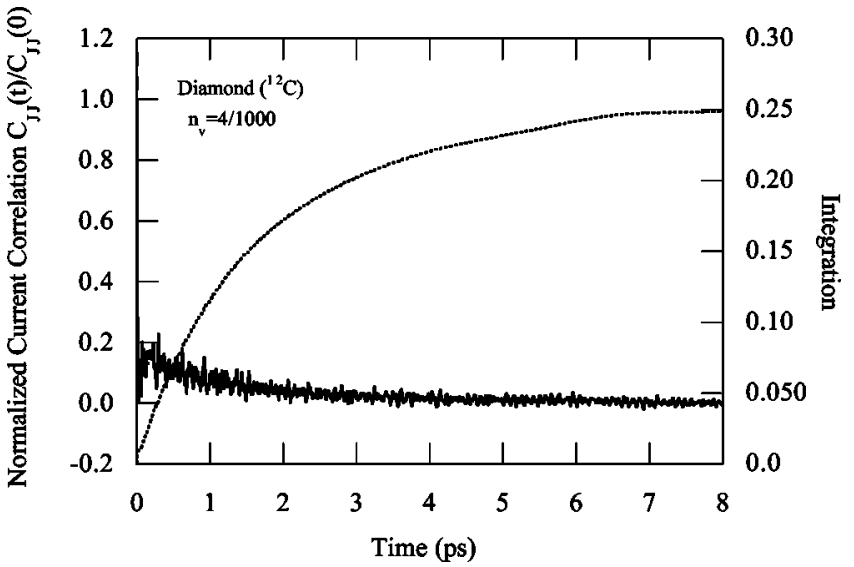

FIG. 5. The heat current correlation function for the system with a concentration $n_{v}=0.004$ vacancy. The exponential fit to the data is shown by dashed line (almost invisible under the heat correlation function). The time integrated curve is shown by the dotted line.

thermal conductivity. This indicates that optical phonons have little effect on the values of the thermal conductivity (see the $A_{o} \tau_{o}$ value in Table I). Figure 5 depicts the heat current correlation function for the case of four vacancies per 1000 atoms. Since the heat current correlation function is well converged, both direct integration and exponential fitting give the same results for the thermal conductivity. This justifies the use of exponential fitting in the previous calculations, since only the portion of converged correlation functions were used to carry out the fitting.

\section{Mass dependence of thermal conductivity}

In the classical limit, the thermal conductivity is expected to depend inversely on the square root on atomic mass. This is because velocity of sound scales as $1 / \sqrt{M}$ whereas the mean free path and specific heat do not change appreciably with mass in the classical limit. Figure 6 shows that the calculated thermal conductivity as a function of the atomic mass behaves as $1 / \sqrt{M}$.

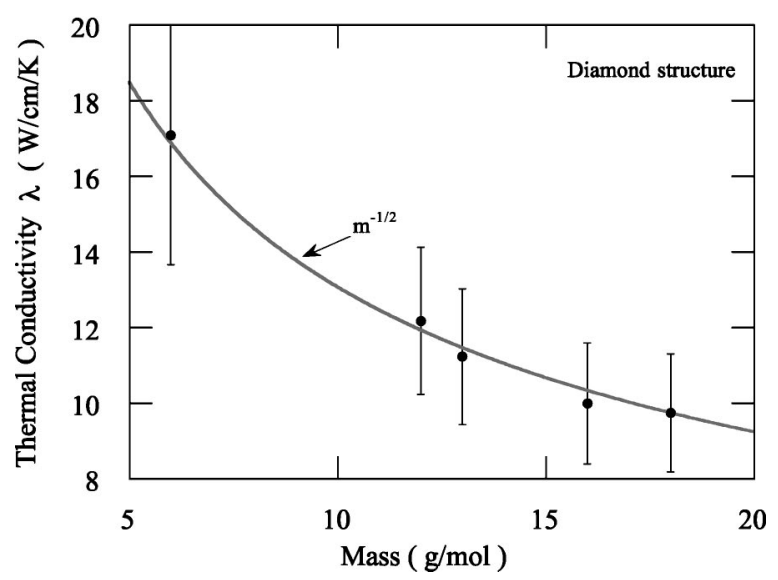

FIG. 6. The dependence of the calculated thermal conductivities (pure systems) on atomic mass. The solid line is the fit to the phenomenological relation of $A / \sqrt{M}$. 


\section{Binary alloys}

A primary motivation in developing techniques to use MD simulations for calculating thermal conductivity is to predict the thermal properties of electronic devices based of thin films of heterostructures (e.g., the GaN/AlN/SiC/Si structure for high quality GaN films). To study the effects on thermal conductivity of mass mismatches in these systems, we chose to use the diamond FF but to consider equal numbers of atoms with light and heavy masses $M_{l}$ and $M_{h}$. We kept $M_{l}+M_{h}=24 \mathrm{u}$ so that the macroscopic density remains constant, leading to the same velocity of sound (using the same FF leads to the same elastic constants). In the classical limit the specific heat will also remain constant in Eq. (47). In this case, the variation of thermal conductivities should depend mainly on the variation of phonon mean free path.

\section{The $M_{l} M_{h}$ sphalerite (B3 cubic) system}

First we consider the case in which the $M_{l}$ and $M_{h}$ alternate as in the cubic sphalerite or zinc blende structure (B3). The heat current correlation function at short times for $M_{l} / M_{h}=11 / 13$ is shown in Fig. 7(a). A striking feature here is that the heat current correlation function has a regular high frequency oscillation absent in the single component systems. This is due to the relative oscillation between bonded atoms with different mass. The lighter mass atoms move with a higher average velocity than the heavier mass atoms, giving a high amplitude heat current oscillation. When the two masses are the same, the heat current due to this (optical) oscillation tends to cancel, so that no such heat current oscillations are observed in single component systems (see the discussion in Sec. IV B). The Fourier transformation of the correlation function, Fig. 7(c), shows a strong peak at $1342 \mathrm{~cm}^{-1}$ that represents this oscillation frequency. Figure 8 shows how this frequency depends on the reduced mass, $\mu=M_{l} M_{h} /\left(M_{l}+M_{h}\right)$. We see that

$$
\omega(\mu)=A \sqrt{\frac{1}{\mu}},
$$

where $A=3353.3 \mathrm{~cm}^{-1}(\mathrm{~g} / \mathrm{mol})^{1 / 2}$. This compares to a calculated frequency ${ }^{29}$ for $\Gamma_{15}$ of $1288 \mathrm{~cm}^{-1}$.

The oscillation in the original correlation function Fig. 7(a) makes it difficult to do either a direct integration or an exponential fitting. Therefore, to extract the underlying behavior of the correlation function, we used a square low pass Fourier filter to remove the oscillations above $6 \mathrm{THz}$ $\left(200 \mathrm{~cm}^{-1}\right)$, since such modes do not contribute to the thermal conductivity. This leads to the correlation function in Fig. 7(b), which was fitted to the double exponential functions. The validity was checked by the same procedure as for the single-component system. The thermal conductivity was calculated using Eq. (46), leading to the thermal conductivities as a function of the reduced mass depicted in Fig. 9.

Figure 9 shows that the thermal conductivity drops sharply when the single-component system is changed into a binary system. Thus, $\lambda$ drops by $37.1 \%$ when the singlecomponent ${ }^{12} \mathrm{C}$ system is changed to the $11 / 13$ binary system. This drop implies a significant increase in the phonon scattering for the binary system as compared to a singlecomponent system. The result is not so surprising when we

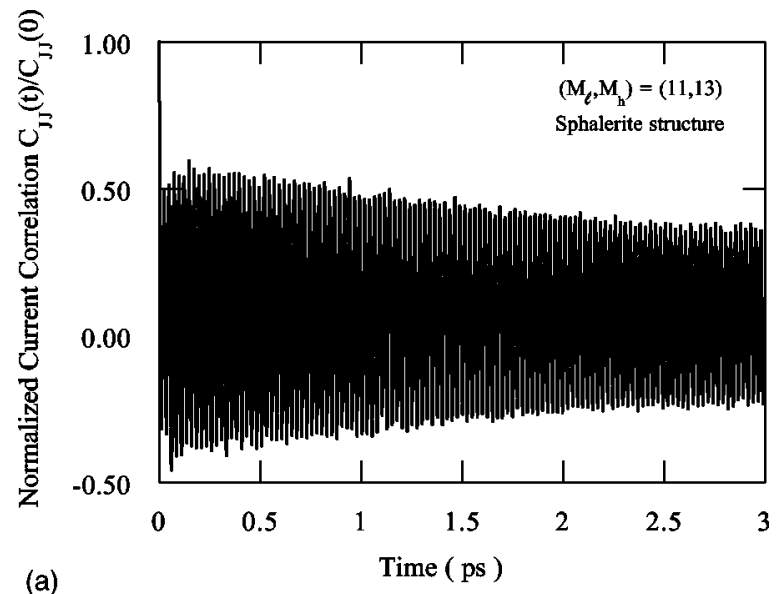

(a)

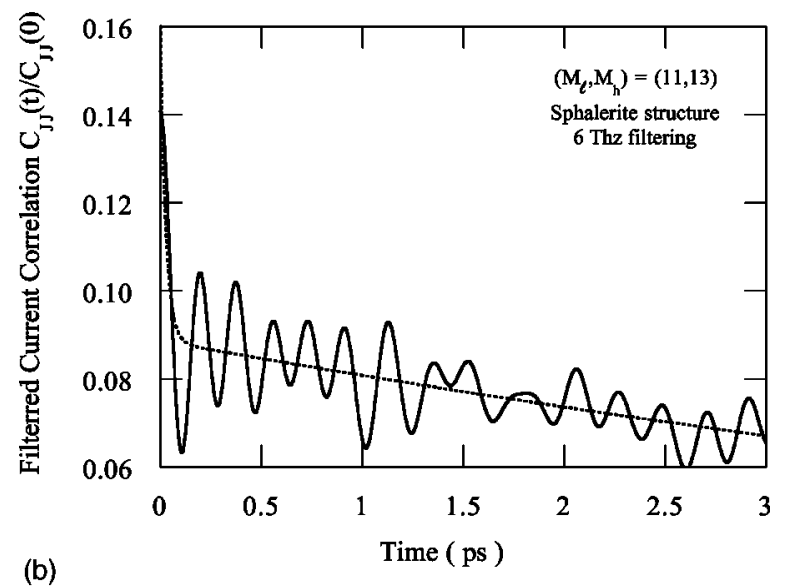

(b)

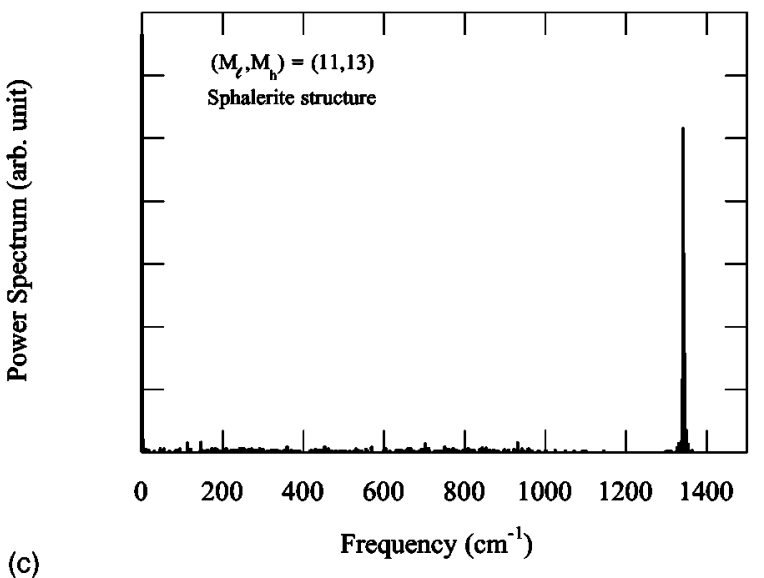

FIG. 7. (a) The heat current correlation function for $\left(M_{l}, M_{h}\right)=(11,13)$ sphalerite. (b) The heat current correlation function from (a) after Fourier low pass filtering using $\nu_{\max }=6(\mathrm{THz})$. The dotted line is the fitted double exponential fitting. (c) Power spectrum of (a). The peak at $1342 \mathrm{~cm}^{-1}$ is interpreted as the optimal vibrational mode $\left(\Gamma_{15}\right)$.

consider that just $1.1 \%$ of ${ }^{13} \mathrm{C}$ in the natural diamond leads to a thermal conductivity $31 \%$ lower than for pure ${ }^{12} \mathrm{C}$ diamond crystal.

Figure 9 shows two regions. Below $\mu=5.625\left(M_{l} / M_{h}\right.$ $=9 / 15)$ the $\lambda$ is linear in $\mu$, leading to $\lambda=\lambda(o)+A \mu$ with $A=0.72$. Whereas for $\mu$ above $5.958\left(M_{l} / M_{h}=11 / 13\right) \lambda$ is linear in $\mu$ with $\lambda=\lambda(o)+B \mu$ where $B=117.5$. We do not have an explanation for this sharp transition. 


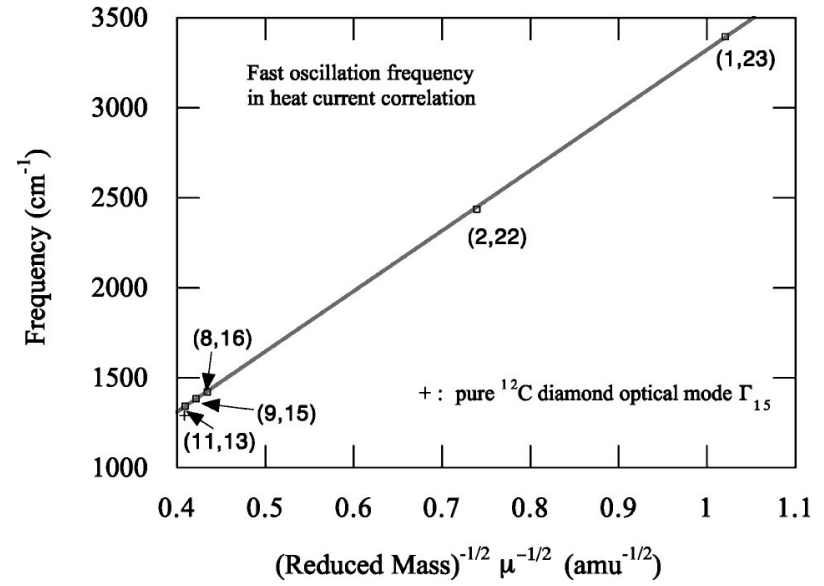

FIG. 8. The characteristic frequencies of $\left(M_{l}, M_{h}\right)$ sphalerite extracted from the Fourier transform of the heat current correlation function. The composition of $\left(M_{l}, M_{h}\right)$ is labeled. The solid line is the linear fit [with $A$ $\left.=3353.3 \mathrm{~cm}^{-1} /(\mathrm{u})^{1 / 2}\right]$. The plus symbol indicates the frequency of the $\Gamma_{15}$ vibration in pure ${ }^{12} \mathrm{C}$ diamond.

\section{Layered alloys of diamond}

It is of interest to understand how the thermal conductivity depends on thin layers with different masses and force constants (e.g., GaN/AlN/SiC/Si). Here size confinement and interface effects can lead to thermal conductivities for thin layer structures quite different from the uniform bulk systems. The phonon mean free path of a thin layer structure is limited by the scattering on the interfaces. To study such systems, we formed layered structures using two masses $\left(M_{l}\right.$ and $M_{h}$ ). We considered $M_{l} / M_{h}=4 / 20$ to match the mass ratio in a $\mathrm{GaN}$ semiconductor. In the limit of an alternating mass for each atomic layer, we obtain exactly the sphalerite crystal structure. As indicated in Fig. 9, the $(4,20)$ case leads to $\lambda=5.42 \mathrm{~W} / \mathrm{cm} / \mathrm{K}$ compared to $\lambda=12.2 \mathrm{~W} / \mathrm{cm} / \mathrm{K}$ for pure ${ }^{12} \mathrm{C}$.

Increasing the thickness of each layer while keeping the total number of $M_{l}$ and $M_{h}$ atoms fixed leads to variation in the thermal conductivity. From mass dependence studies of perfect crystals (see Sec. IV B 3), we expect that

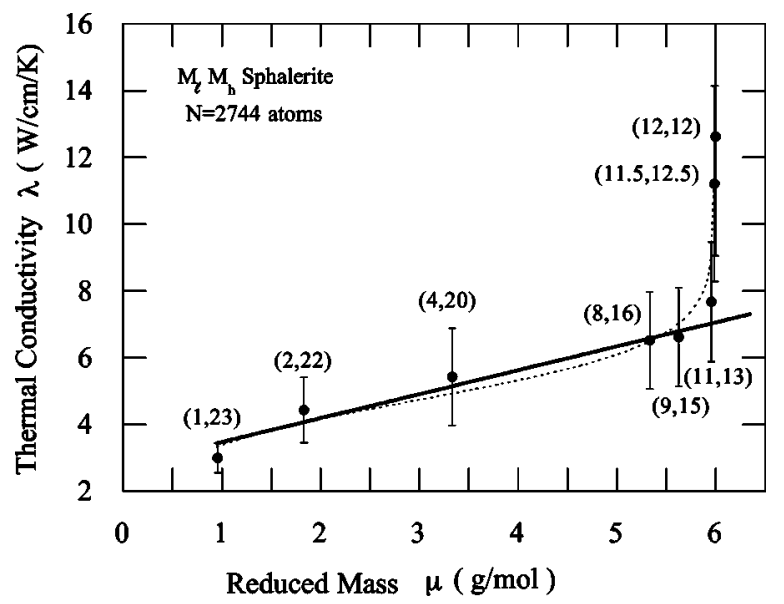

FIG. 9. The thermal conductivity of $\left(M_{l}, M_{h}\right)$ sphalerite as a function of reduced mass, $\mu=M_{l} M_{h} /\left(M_{l}+M_{h}\right)$. The solid line is the linear fit where the dashed line is a smooth function fit.

$$
\begin{aligned}
\lambda_{l} & =\frac{\sqrt{M_{c}} \lambda_{c}}{\sqrt{M_{l}}}, \\
\lambda_{h} & =\frac{\sqrt{M_{c}} \lambda_{c}}{\sqrt{M_{h}}} .
\end{aligned}
$$

For a macroscopic system, we obtain,

$$
\lambda_{\perp}^{-1}=\frac{1}{2}\left(\lambda_{l}^{-1}+\lambda_{h}^{-1}\right), \quad \lambda_{\|}=\frac{1}{2}\left(\lambda_{l}+\lambda_{h}\right) .
$$

Combining Eqs. (56), (54), and (55), and ignoring the effects of interfaces, we would expect that the thermal conductivity of layered structure to be

$$
\begin{aligned}
& F_{\|}=\frac{\lambda_{\|}}{\lambda_{c}}=\frac{\sqrt{M_{c}}\left(\sqrt{M_{l}}+\sqrt{M_{h}}\right)}{2 \sqrt{M_{l} M_{h}},} \\
& F_{\perp}=\frac{\lambda_{\perp}}{\lambda_{c}}=\frac{2 \sqrt{M_{c}}}{\sqrt{M_{l}}+\sqrt{M_{h}}} .
\end{aligned}
$$

Taking $M_{l}=4, M_{h}=20$, and $M_{c}=12$, we obtain $F_{\|}=1.45$ and $F_{\perp}=1.07$ for the thermal conductivity of layered structure in the continuum limit. These results differ dramatically from simulation results which leads to $F_{\|}=0.42$ and $F_{\perp}$ $=0.0034$ for the $l^{12} h^{12}$ case. This disagreement shows that there are clear size effects. Such size effects can be explained by the fact that the phonon mean free path is larger than the layer dimensions, making the impedance mismatch at the interfaces important. Such size effects in the thermal conductivity for nanoscale dimensions may have significant impact on the design of nanoscale devices (where energy must be rapidly dissipated).

To provide a relationship between the microscopic and continuum description, we introduce a boundary scattering process with a scattering length $D^{\perp, \|}$ proportional to the layer thickness $l$,

$$
D_{\perp, \|}=l / \alpha^{\perp, \|},
$$

where $\alpha^{\perp, \|}$ is the scale constant that accounts for the effects in scattering in the perpendicular or parallel direction due to the level of interface mismatch. The symbols $\perp, \|$ denote the directionality with respect to layer orientation. We expect that $\alpha^{\perp} \leqslant 1$, but that $\alpha^{\perp} \rightarrow 1$ for very distinct materials due to increasing boundary scattering of phonons. We expect that $\alpha^{\|}$is very small since the layered structure should have little effect on the parallel direction.

In the independent scattering approximation, we write the total phonon mean free path $L_{\text {tot }}$ as

$$
L_{\mathrm{tot}}^{-1}=(1-s)\left(L^{-1}+D_{l}^{-1}+D_{h}^{-1}\right)+s L_{l h}^{-1} .
$$

Here $L$ is the effective scattering length for pure systems and $L_{l h}$ is the value for the sphalerite light-heavy systems. $D_{l}$ and $D_{h}$ are the characteristic scattering lengths for the light and heavy portions of the system. Our simulations have $l$ as half of the periodicity for the perpendicular direction (i.e., actual layer thickness). $s$ is an order parameter indicating how much the system differs from a pure sphalerite structure: For pure sphalerite structure $s=1$, and for completely layered structure $s=0$. Using Eq. (60), we can write the thermal conductivity for layered systems as 


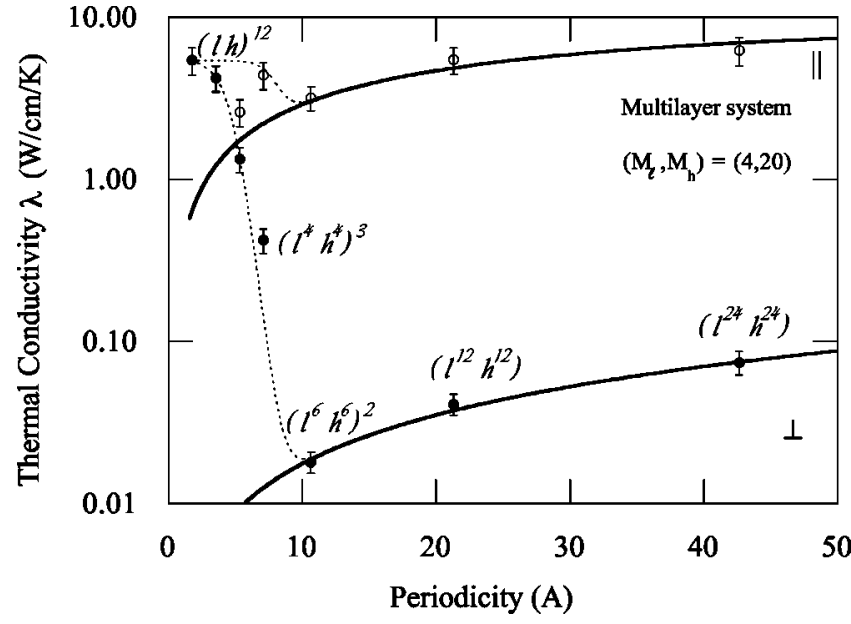

FIG. 10. The thermal conductivity for $M_{l} / M_{h}$ layered systems (the distribution of atoms in the layers is shown). The closed circles indicate the thermal conductivity in the direction perpendicular to the layers, and the open circles are for the ones parallel to the layers. The solid lines are calculated from Eqs. (61) and (62) with $s=0$ and $\alpha^{\perp, \|}=1$. The dashed lines are calculated also from Eqs. (61) and (62) but with $s \neq 0$ [taken $s$ in the form of Eq. (65)].

$$
\begin{aligned}
& \lambda_{\perp}=\lambda_{c} L^{-1}\left[s L_{l h}^{-1}+(1-s)\left(L^{-1}+D_{l, \perp}^{-1}+D_{h, \perp}^{-1}\right)\right]^{-1}, \\
& \lambda_{\|}=\lambda_{c} L^{-1}\left[s L_{l h}^{-1}+(1-s)\left(L^{-1}+D_{l, \|}^{-1}+D_{h, \|}^{-1}\right)\right]^{-1} .
\end{aligned}
$$

Note that Eqs. (61) and (62) are valid for systems with the same density, in other words, with the same heat capacity and velocity of sound in the classical limit. In pure light or heavy systems, $\lambda_{c}$ will have to be modified accordingly. At the sphalerite limit, Eqs. (61) and (62) lead to the thermal conductivity of the binary composite,

$$
\lambda=\lambda_{\perp}=\lambda_{\|}=\lambda_{c} L^{-1} L_{l h}=\lambda_{l h} .
$$

In a previous section, we obtained $L=174 \mathrm{~nm}$. Thus, we can easily deduce the phonon mean free path $L_{l h}$ from Eq. (63),

$$
L_{l h}=77 \mathrm{~nm} \text {. }
$$

The case of $s=0$ in Eqs. (61) and (62) (a system far from the sphalerite limit) leads to the solid line in Fig. 10, which uses $\alpha^{\perp}=1$ and $\alpha^{\|}=0.004$. This suggests that the phonon scattering at a perpendicular interface is $\sim 250$ times larger than for a parallel interfaces. The closed circles show the simulated conductivities calculated from the simulations in the direction perpendicular to the layers. We see that for layer thicknesses above 6 atomic layers $(\sim 10 \AA)$, the thermal conductivities agree well with the continuum theory. This suggests that for this system $s=0$ for layers thicker than 10 $\AA$. For layers thinner than $10 \AA$, the thermal conductivity jumps back to a value $\sim 100$ times larger, approaching the sphalerite value at the limit of $s=1$.

This behavior suggests that increasing thicknesses of 10 $\AA$ in nanoscale composites can have a very dramatic decrease in the thermal conductivity. Equations (61) and (62) in the simulation results leads to $s_{1}=1.0, s_{2}=0.9997, s_{3}$ $=0.9941, s_{4}=0.9733$, and $s_{6}=0$, where the subscripts indicate the number of layers. We then described this order parameter in an approximate fitted form to the

$$
s=\frac{1+\cos [0.01138(x-1.778)]}{2\left[1+\exp \left(\frac{x-8.505}{0.5896}\right)\right]},
$$

where $x$ is the periodic length. In terms of layer numbers, Eq. (65) becomes

$$
s=\frac{1+\cos \left[0.01280\left(n_{\perp}-2\right)\right]}{2\left[1+\exp \left(\frac{n_{\perp}-9.567}{0.6632}\right)\right]},
$$

where $n_{\perp}$ is the number of continuous atomic layers having the same mass.

\section{DISCUSSION}

We showed that MD simulations can be used to predict the thermal conductivity of crystalline systems including defects. The numerical results verify our expectation that the heat current correlation function at short times can be used to extract the overall decay constants.

We investigated the quantum correction to the classical thermal conductivity based on the relationship between the quantum correlation function and the classical correlation function. We find that for harmonic systems linearly coupled to a harmonic bath the thermal conduction calculated from the classical correlation function is the same as that calculated from the quantum correlation function. The quantum corrections to the thermal conductivity for a real system come only in higher orders of anharmonicity. Since our MD simulations use a FF which describes anharmonicity, the classical correlation functions have the correct mode coupling in the classical limit. Therefore, Eq. (28) can be thought of as a reasonable approximation for anharmonic systems. This is due to the cancellation of the prefactor $2 \tanh (\beta \hbar \omega / 2) /(\beta \hbar \omega)$ in Eq. (27). Both analytic theory and the MD simulations indicate that no additional quantum corrections are necessary for temperatures even far below the Debye temperature. This requires that we can neglect the pure quantum effects not represented by the harmonic mapping approximation [i.e., Eqs. (28) and (29)]. In cases for which quantum effects must be included such as tunneling and anharmonicity effects in the prefactor for these equations, Eqs. (28) and (29) must be modified. This criteria does not require that the system temperature be higher than the Debye temperature. In fact, quantum effects depending on anharmonic corrections usually become less important for temperatures lower than the Debye temperature. That is, they lead to corrections on top of the quantum harmonic correction, which is easily done. This result is important because it extends the region where classical MD can be applied to calculate thermal conductivity of materials.

The numerical results for diamond-based crystals show that classical MD simulations can provide useful results for the thermal conductivity. In particular, it accounts for the effect of isotopic substitution and vacancies. Thus, classical methods should be useful in describing the thermal conductivities of novel nanometer scale electronic and mechanical systems. The small size of such systems makes experimental measurement quite challenging. The methods outlined here 
suggest a practical approach to using classical calculations to study the thermal properties of such systems.

Although our simulation results for a natural diamond are $60 \%$ lower than the experimental values, we believe that this is mainly because the Brenner FF used in the simulations leads to anharmonicity that is far too large. We believe that more accurate FF will lead to accurate results for these classical methods. Thus, previous calculations ${ }^{40}$ on the thermal conductivity of $\mathrm{C}_{60}$ crystal using a more accurate $\mathrm{FF}$ gave results within experimental accuracy.

\section{ACKNOWLEDGMENTS}

This research was initiated with funds from a grant from NASA (Computational Nanotechnology) and continued with funds from a grant from DOE-ASCI-ASAP. The facilities of the MSC are also supported by grants from BP Amoco, NSF (CHE 95-12279), ARO-MURI, ARO-DURIP, AROASSERT, Beckman Institute, Seiko-Epson, Exxon, AveryDennison Corp., Chevron Corp., Dow, 3M, and Asahi Chemical.

${ }^{1}$ P. Stachowiak, V. V. Sumarokov, J. Mucha, and A. Jezowski, Phys. Rev. B 58, 2380 (1998).

${ }^{2}$ D. J. Evans, Phys. Lett. 91A, 457 (1982).

${ }^{3}$ D. Macgowan and D. J. Evans, Phys. Lett. A 117, 414 (1986).

${ }^{4}$ P. J. Davis and D. J. Evans, J. Chem. Phys. 103, 4261 (1995).

${ }^{5}$ S. Walkauskas, D. Broido, K. Kempa, and T. Reinecke, J. Appl. Phys. 85, 2579 (1999).

${ }^{6}$ A. Balandin and K. Wang, Phys. Rev. B 58, 1544 (1998).

${ }^{7}$ V. L. Gurevich, Transport in Phonon Systems (North-Holland, Amsterdam, 1986).

${ }^{8}$ M. J. Gillan, Phys. Scr., T T39, 362 (1991).

${ }^{9}$ G. V. Paolini, P. J. Lindan, and J. H. Harding, J. Chem. Phys. 106, 3681 (1997).

${ }^{10}$ J. Li, L. Porter, and S. Yip, J. Nucl. Mater. 255, 139 (1998).

${ }^{11}$ V. V. Murashov, J. Phys.: Condens. Matter 11, 1261 (1999).

${ }^{12}$ S. Volz and G. Chen, Physica B 263-264, 709 (1999).

${ }^{13}$ S. G. Volz and G. Chen, Appl. Phys. Lett. 75, 2056 (1999).

${ }^{14}$ S. G. Volz and G. Chen, Phys. Rev. B 61, 2651 (2000).
${ }^{15}$ R. Kubo, M. Toda, and N. Hashitsume, Statistical Physics (Springer, Berlin, 1985), Vol. II.

${ }^{16}$ G. D. Mahan, Many-Particle Physics (Plenum, New York, 1993).

${ }^{17}$ R. Berman, Phys. Rev. B 45, 5726 (1992).

${ }^{18}$ D. Mozelli, Chem. Phys. Carbon 24, 45 (1994).

${ }^{19}$ B. J. Berne and G. D. Harp, Adv. Chem. Phys. 17, 63 (1970).

${ }^{20}$ P. Schofield, Phys. Rev. Lett. 4, 39 (1960).

${ }^{21}$ P. A. Egelstaff and P. Schofield, Nucl. Sci. Eng. 12, 260 (1962).

${ }^{22}$ P. A. Egelstaff, Adv. Phys. 11, 203 (1962).

${ }^{23}$ J. S. Bader and B. J. Berne, J. Chem. Phys. 100, 8359 (1994)

${ }^{24}$ J. Cao and G. A. Voth, J. Chem. Phys. 100, 5106 (1994).

${ }^{25}$ R. D. Feynman and A. R. Hibbs, Quantum Mechanics and Path Integrals (McGraw-Hill, New York, 1965).

${ }^{26}$ D. W. Brenner, Phys. Rev. B 42, 9458 (1990).

${ }^{27}$ D. A. McQuarrie, Statistical Mechanics (Harper \& Row, New York, 1976).

${ }^{28}$ J. M. Ziman, Principles of the Theory of Solids (Cambridge University Press, Cambridge, 1972).

${ }^{29}$ P. H. Berens, D. H. J. Mackay, G. M. White, and K. R. Wilson, J. Chem. Phys. 79, 2375 (1983).

${ }^{30}$ CRC Handbook of Chemistry and Physics, 71st ed. (CRC Press, Boca Raton, FL, 1990).

${ }^{31}$ T. R. Anthony, W. F. Banholzer, J. F. Fleischer, L. Wei, P. K. Kuo, R. L. Thomas, and R. W. Pryor, Phys. Rev. B 42, 1104 (1990).

${ }^{32}$ T. R. Anthony, W. F. Banholzer, and J. F. Fleischer, US Patent No. 5310447 (May 10, 1994), Single-crystal diamond of very high thermal conductivity.

${ }^{33}$ W. F. Banholzer and T. R. Anthony, Thin Solid Films 212, 1 (1992).

${ }^{34}$ L. Wei, P. K. Kuo, R. L. Thomas, T. R. Anthony, and W. F. Banholzer, Phys. Rev. Lett. 70, 3764 (1993).

${ }^{35}$ J. R. Olson, R. O. Pohl, J. W. Vandersande, A. Zoltan, T. R. Anthony, and W. F. Banholzer, Phys. Rev. B 47, 14850 (1993).

${ }^{36}$ The value is calculated numerically using finite displacements of 0.01 and $0.02 \AA$ in the $\{100\}$ axis direction for cubic diamond crystal. Using the same displacements in the $\{111\}$ direction leads to $1302 \mathrm{~cm}^{-1}$.

${ }^{37}$ This value is calculated from the least-squares fit of the relation $3 \beta T$ $+C=\ln V$ for the system's volume and temperature of 2744 atoms at zero external pressure, where $\beta=(1 / 3 V)(\partial V / \partial T)_{p}$ and $C$ is a constant. The calculated result for $\beta$ is consistent with the value calculated from fluctuation formula, $\langle\delta V \delta H\rangle_{N P T}=3 k_{B} T^{2} V \beta$.

${ }^{38}$ CRC Handbook of Chemistry and Physics, 76th ed. (CRC Press, Boca Raton, FL, 1995).

${ }^{39}$ J. M. Ziman, Electrons and Phonons (Clarendon, Oxford, 1967).

${ }^{40}$ J. Che, T. Cagin, and W. A. Goddard III, Nanotechnology 10, 263 (1999). 